\title{
Performance analysis of multiplexing and error control scheme for body area networks
}

\author{
Kento Takabayashi ${ }^{1 *}$, Hirokazu Tanaka ${ }^{2}$, Chika Sugimoto ${ }^{1}$ and Ryuji Kohno ${ }^{1}$
}

\begin{abstract}
In this paper, we theoretically analyze the performance of a multiplexing and error control scheme for body area networks. In our previous work, we proposed a quality of service (QoS) control optimization method that achieves optimal QoS control by introducing a multiplexing scheme over the media access control (MAC) layer. This multiplexing scheme combines Weldon-based hybrid automatic repeat request (ARQ) with a decomposable error-correcting code. In this paper, we present a theoretical analysis of our proposed scheme as an extension of our previous work. In this proposed system, the decomposable code which has simpler structure is utilized. We then show that our proposed multiplexing layer can achieve optimal performance at $E_{s} / N_{0}=3,5$, and $6 \mathrm{~dB}$ in the additive white Gaussian noise (AWGN) channel and at $E_{s} / N_{0}=8,11$, and $14 \mathrm{~dB}$ in the Rayleigh fading channel by arbitrarily selecting parameters for the error-correcting code and hybrid ARQ. Then, we show that the proposed system obtains over $1.2 \mathrm{~dB}$ gain in the AWGN channel and over $4.2 \mathrm{~dB}$ gain in the Rayleigh fading channel than IEEE802.15.6 in the optional pattern.
\end{abstract}

Keywords: Wearable sensor, Body area network, QoS control, Multiplexing

\section{Introduction}

Health monitoring systems that employ wearable vital sensors and wireless communication have recently received significant attention [1-16]. It is expected that wearable sensors will pave the way for a new era of tele-healthcare that may include continuous monitoring of physical conditions and the detection of serious consequences. In the field of health-monitoring systems, the body area network (BAN) is a key technology $[1-4,10-16]$. Its standardization activities have been carried out extensively [17-19]. To realize smaller sensor devices and longer battery life, the following technical requirements should be considered.

- Ultra-low power consumption Although this has been considered in the above standards, substantially lower power-consuming media access control (MAC) and physical layer (PHY) technologies are required.

- Coexistence with other networks

The 2.4-GHz industrial, scientific, and medical (ISM) band is globally assigned for common use in local area network (LAN) and personal area network (PAN) devices. This frequency band is potentially a good candidate for BANs; however, when using this band, interference from other systems must be taken into consideration.

- Optimal quality of service (QoS) control A wearable vital sensor can connect various types of sensors, with the data rate of such sensors varying widely. Further, their respective allowable delay times depend on their application. Therefore, optimal QoS control for input data is an important factor in transmitting sensor data.

From the above requirements, we have proposed an optimal QoS control scheme that employs a multiplexing layer and a decomposable error control coding scheme [20-22]. In [20], we proposed a QoS control 
scheme using Weldon-based hybrid automatic repeat request (ARQ) [23] with Reed-Muller codes. However, the error-correcting capability could not be increased effectively because of characteristics of Reed-Muller codes which we selected in [20]. So, we conducted simulations to evaluate the performance of the proposed system utilizing Weldon's ARQ with decomposable codes based on punctured convolutional codes in the additive white Gaussian noise (AWGN) channel [21] and a wearable BAN channel model [22] by comparing it with IEEE 802.15.6. Assuming that the wearable sensor device has multiple sensors, each sensor's input data are transmitted through a common MAC and PHY layer [21, 22].

Because we evaluated the effectiveness of our proposed scheme only via simulation in our previous work, in this paper, we present a theoretical analysis of our proposed scheme in [21] and [22] under the AWGN channel and the Rayleigh fading channel as an extension of our previous work. We have not evaluated and analyzed the case of the Rayleigh fading channel in our previous work. Then, in this proposed system, the structure of our decomposable code is modified to be much simpler than that in our previous work [21, 22] in order to analyze it more easily. In general, an evaluation of ARQ requires a large number of simulation trials to fully evaluate the given system; however, we can specifically evaluate the throughput in a stable state by sound theoretical analysis. More specifically, we can easily obtain detailed characteristics of our proposed scheme by this formulization. We derived a lower bound on throughput performance and an upper bound on residual bit error rate in the AWGN channel and the Rayleigh fading channel. In addition, we investigated QoS parameters to further optimize our system. For example, we identified the optimum parameters according to various policies, as shown in Tables 3 and 4. Figure 8 shows the results of our throughput optimization by using the parameters of Table 4.

The remainder of this paper is organized as follows. In Section 2, we briefly review the system model of our proposed scheme. Section 3 explains the theoretical analysis of our proposed scheme under the AWGN channel and the Rayleigh fading channel. Performance evaluations by theoretical analysis and simulations are presented in Section 4. Finally, we conclude our paper and provide directions for future work in Section 5.

\section{System model}

\subsection{System concept}

Figure 1 shows the overall concept of our proposed system. In general, different types of data are input and multiplexed at a single sensor device [19]. Here, we assume that the different types of data input and multiplexed have potentially different QoS requirements. Given the varying QoS requirements, the different types of data have different priorities. These data are multiplexed and transmitted from a sensor (i.e., a wireless body area network (WBAN) node) to a WBAN coordinator.

Figure 2 shows the system model. The transmitter consists of a multiplexing module, a MAC module, and a PHY module. The multiplexing module controls the different types of QoS requirements according to the following priorities. First, several data are added to user priorities. Then, the header, which includes user priority information (e.g., latency, rate of error control coding,

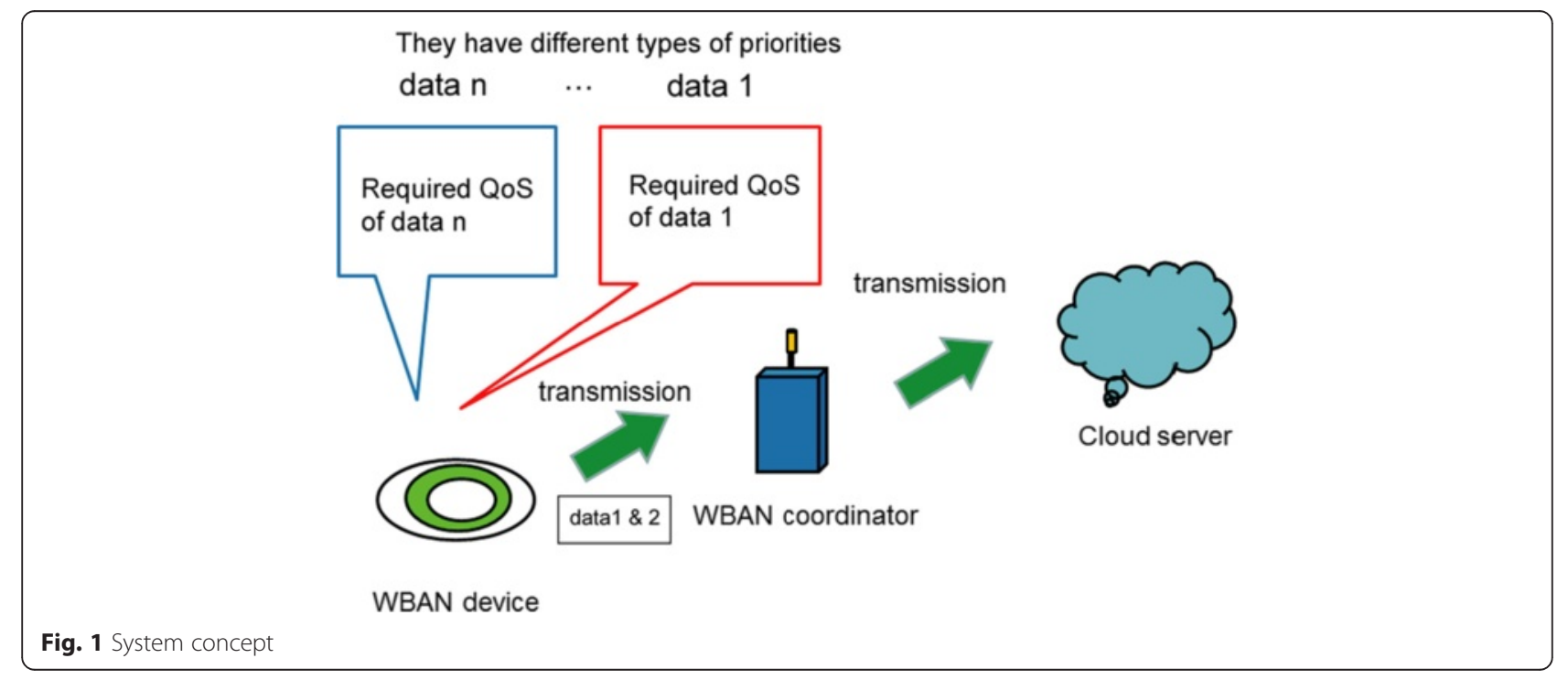




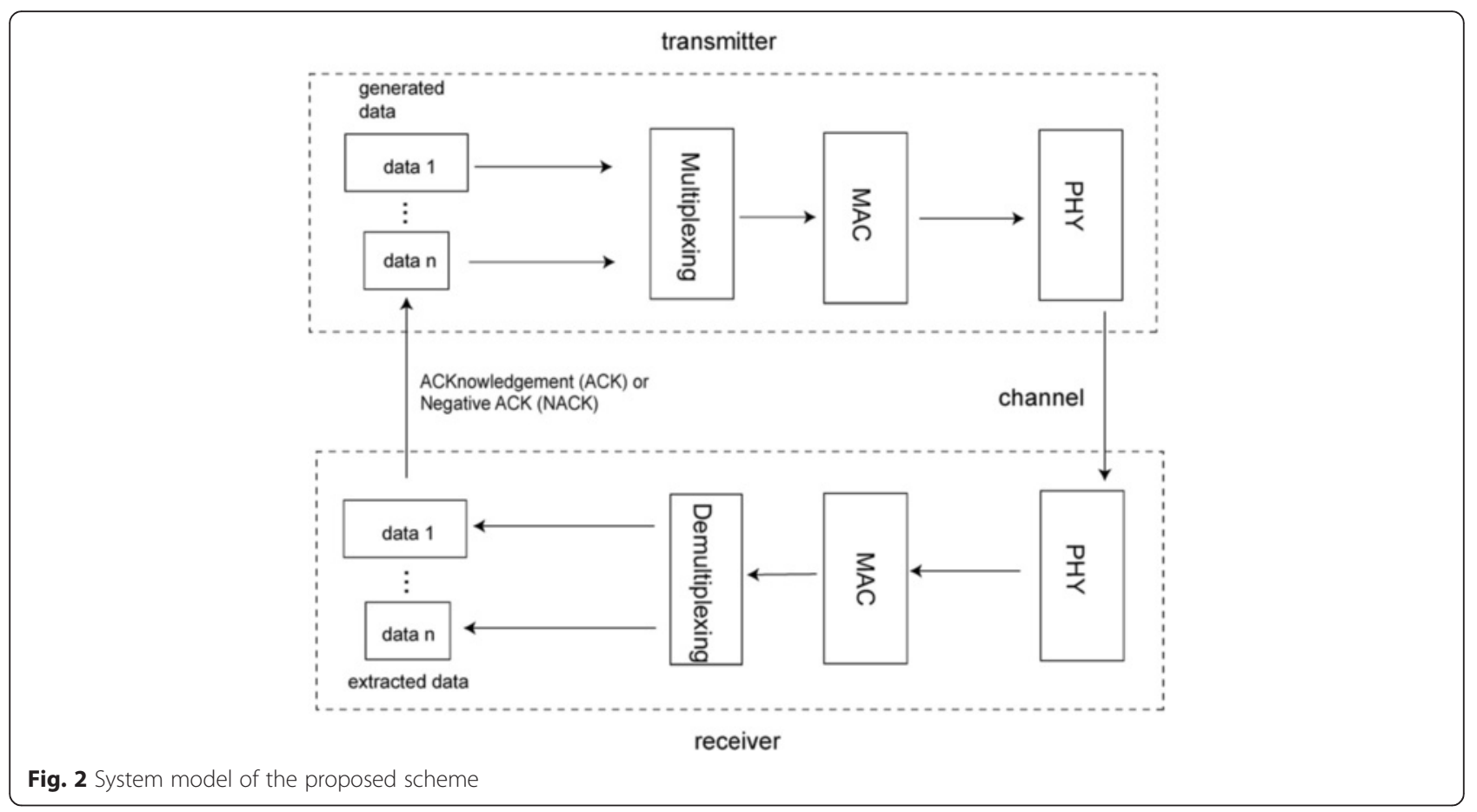

\begin{tabular}{|c|c|c|c|c|c|c|c|c|c|c|}
\hline information $m$ & $m_{1}$ & $m_{2}$ & & $m_{3}$ & $m_{4}$ & & $m_{5}$ & $m_{6}$ & $m_{7}$ & $m_{8}$ \\
\hline output $u_{1}$ & $u_{11}$ & $u_{12}$ & & $u_{13}$ & $u_{14}$ & & $u_{15}$ & $u_{16}$ & $u_{17}$ & $u_{18}$ \\
\hline output $u_{2}$ & $u_{21}$ & $u_{22}$ & & $u_{23}$ & $u_{24}$ & & $u_{25}$ & $u_{26}$ & $u_{27}$ & $u_{28}$ \\
\hline \multicolumn{11}{|c|}{ punctured matrix } \\
\hline & & 1 & 0 & 1 & 1 & 0 & 1] & & & \\
\hline & & 0 & 1 & 0 & 0 & 1 & 0] & & & \\
\hline
\end{tabular}

\begin{tabular}{|l|c|c|c|c|c|c|c|c|}
\hline information $m$ & $m_{1}$ & $m_{2}$ & $m_{3}$ & $m_{4}$ & $m_{5}$ & $m_{6}$ & $m_{7}$ & $m_{8}$ \\
\hline output $u_{1}$ & $u_{11}$ & $u_{12}$ & $\times$ & $u_{14}$ & $\times$ & $u_{16}$ & $\times$ & $u_{18}$ \\
output $u_{2}$ & $u_{21}$ & $\times$ & $u_{23}$ & $\times$ & $u_{25}$ & $\times$ & $u_{27}$ & $\times$
\end{tabular}

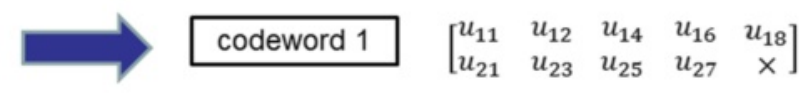

and

\begin{tabular}{|l|c|c|c|c|c|c|c|c|}
\hline information $m$ & $m_{1}$ & $m_{2}$ & $m_{3}$ & $m_{4}$ & $m_{5}$ & $m_{6}$ & $m_{7}$ & $m_{8}$ \\
\hline output $u_{1}$ & $u_{11}$ & $\times$ & $u_{13}$ & $\times$ & $u_{15}$ & $\times$ & $u_{17}$ & $\times$ \\
output $u_{2}$ & $u_{21}$ & $u_{22}$ & $\times$ & $u_{24}$ & $\times$ & $u_{24}$ & $\times$ & $u_{28}$
\end{tabular}

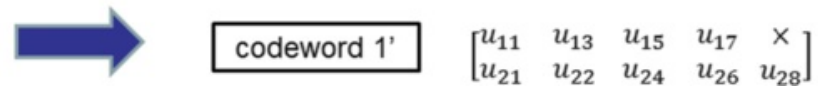

Fig. 3 Punctured convolutional code 
the number of repetitions in ARQ, etc.), is added to the user priorities in the multiplexing layer. Next, the multiplexer controller in the multiplexing layer provides instructions to each data depending on predefined parameters. According to the QoS control signal, the multiplexing layer performs error and delay control. Finally, data with different user priorities are multiplexed and sent to the MAC layer.

In the MAC module, the error control process is performed according to the instructions from the multiplexing module. In the PHY module, this multiplexed data is modulated. In this paper, coherent phase shift keying (PSK) is used for basic analysis. Then, direct sequence spread spectrum (DSSS) is applied to increase robustness against multipath fading and multiuser interference. At the receiver, the transmission operation is processed in the reverse order. Finally, after the process at the demultiplexing module is complete, error detection is performed on the data.

\subsection{Decomposable code}

In our proposed scheme, Weldon's ARQ [23] is employed rather than selective repeat $A R Q$, and decomposable code is employed as error-correction code for hybrid ARQ. The proposed scheme can provide an error control method that satisfies various QoS requirements by coordinating the number of data copies and changing how the decomposable code is combined.

As an example of decomposable code, a punctured convolutional code with constraint length $K$ set to 3 and coding rates $8 / 9,4 / 5,2 / 3$, and $1 / 2$ is used. In [21] and [22], we selected coding rates $7 / 8,5 / 6,3 / 4$, and $1 / 2$. However, those punctured matrices in [21] and [22] are very complicated, and then it is very difficult to analyze its performance in theory. On the other hand, punctured matrices which we select in this paper are quite simple and can be analyzed more easily.

The punctured convolutional code is generated based on the convolutional code with a generator polynomial of $[5,7]$ and coding rate $R=1 / 2$. The punctured matrix of $R=8 / 9$ is shown in Fig. 3. The two patterns of the $R=8 / 9$ punctured codes (codeword 1 and codeword $1^{\prime}$ ) can be generated using this punctured matrix. More specifically, at the first transmission, codeword 1 is sent; then, to increment the code rate of the punctured code, a part of codeword 1' is sent as the second transmission. Figure 4

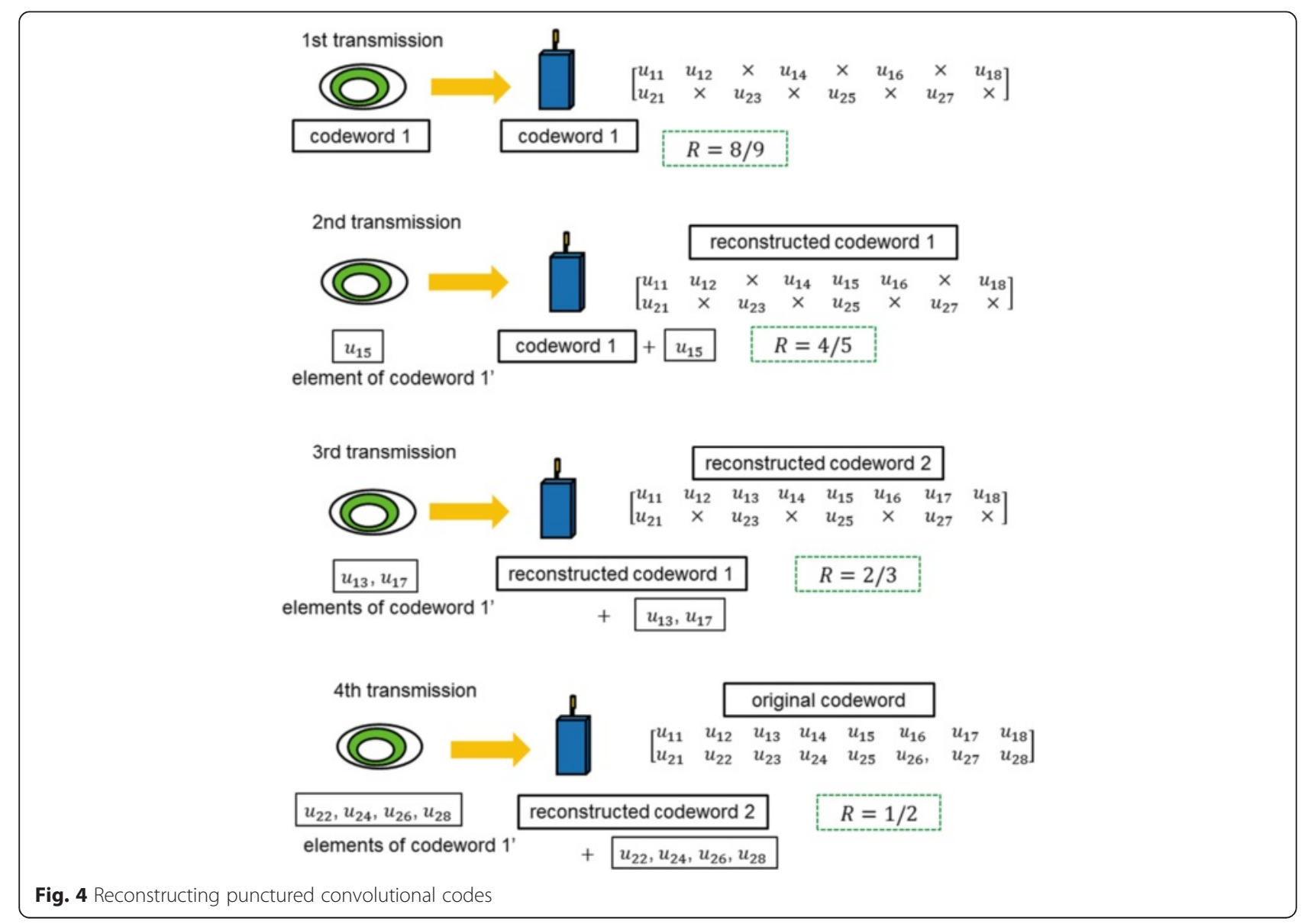


illustrates how to increment redundancies and send the sub-codewords except $u_{11}$ and $u_{21}$ from the first transmission to the fourth transmission. As shown in the figure, these punctured matrices consist of a subset that is a part of the next punctured matrix. In general, at the $i$ th retransmission, reconstructed codewords are decoded as error-correction codes with a coding rate set as follows:

$$
R_{\mathrm{i}}=\left\{\begin{array}{l}
8 / 9(i=0) \\
4 / 5(i=1) \\
2 / 3(i=2) \\
1 / 2(i \geq 3)
\end{array} .\right.
$$

The error-correction capability increases as the coding rate decreases in the order $8 / 9,4 / 5,2 / 3$, and $1 / 2$.

\subsection{Procedure of the proposed scheme}

Figure 5 shows a flowchart of the protocol for our proposed scheme. In the proposed method, retransmission is performed as follows. First, information $m$ is encoded via the punctured convolutional code whose $R=8 / 9$; then, codeword 1 in Fig. 4 is transmitted. If errors are detected, the receiver stores the transmitted codeword 1 and the transmitter re-sends the sub-codeword of codeword $1^{\prime} n_{i}$ times in the case $1 \leq i \leq 3$. At the receiver, a received sub-codeword and stored codeword are combined (Fig. 4), and the reconstructed codeword is decoded. After the third retransmission, codeword 1 is sent $n_{4}$ times and combined with a buffered codeword. If errors are detected, one of $n_{4}$ codeword 1 is buffered in the receiver, and codeword $1^{\prime}$ is transmitted $n_{5}$ times and combined with a stored codeword. After that, codeword 1 and codeword $1^{\prime}$ are sent alternately $n_{i}$ times and stored. When codeword 1 or codeword 1' is buffered, the first one among $n_{i}$ copies is stored. The transmitter repeats these operations until the data is received correctly or the number of retransmissions reaches the predefined maximum number of retransmissions $q$.

\section{Theoretical analysis}

We define normalized throughput $\eta=k_{0} / T$ as the number of total communicated bits and uncoded bits $k_{0}$. The number of total communicated bits $T$ is determined as follows:

$$
\begin{gathered}
T \cong \sum_{i=0}^{q} \prod_{j=0}^{i} P_{j-1}^{n_{j-1}}\left(1-P_{i}^{n_{i}}\right)\left(\sum_{k=0}^{i} n_{k} m_{k}\right)+\sum_{j=0}^{q} n_{j} m_{j} \prod_{j=0}^{q} P_{i}^{n_{i}} \\
\left(* P_{-1} \equiv 1, n_{-1} \equiv 0\right) .
\end{gathered}
$$

Here, $P_{i}$ is the packet error rate (PER), $m_{i}$ is the number of transmitted bits, and $n_{i}$ is the number of copy

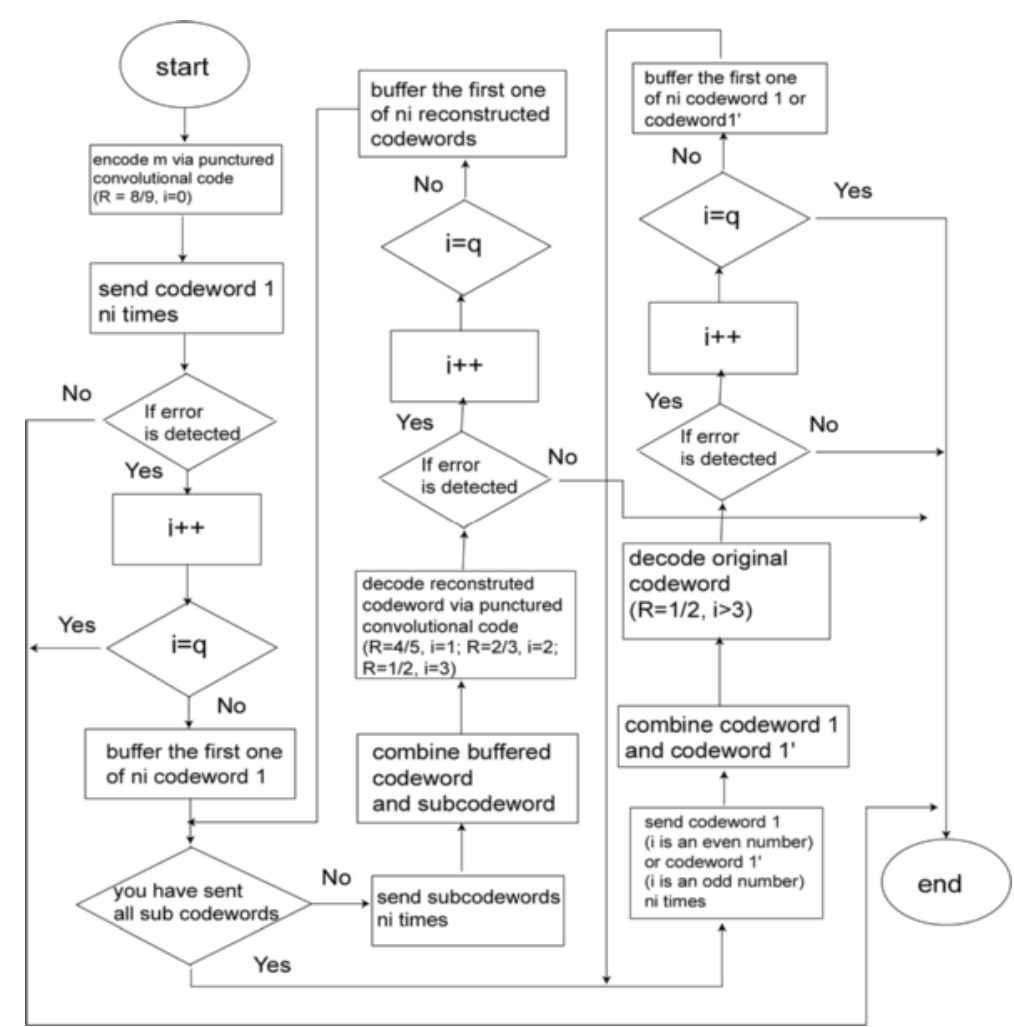

Fig. 5 Flowchart of the proposed ARQ protocol 
blocks of Weldon's ARQ at the $i$ th transmission. Then, $P_{i}$ changes in stages because the received data is decoded by decomposable codes with coding rates varying in order of Eq. 1. Note that throughput $T$ is described as the above approximate equation due to the maximum retransmission limit.

Next, we consider the upper bounds on error probability of the punctured convolutional codes used by the proposed scheme to obtain $P_{i}$. These bounds are obtained from the transfer function $T(D, N)$ of the code, which describes the weight distribution or weight spectrum of the incorrect codewords and the number of bit errors on these paths [24]. The transfer function $T(D, N)$ is expressed as follows:

$$
\begin{aligned}
& \left.T(D, N)\right|_{N=1}=\sum_{d=d_{\text {free }}}^{\infty} a_{d} D^{d} \\
& \left.\frac{d T(D, N)}{d N}\right|_{N=1}=\sum_{d=d_{\text {free }}}^{\infty} c_{d} D^{d} .
\end{aligned}
$$

Here, $d_{\text {free }}$ is the free distance of the code, and $a_{d}$ is the number of incorrect paths or adversaries of the Hamming weight $d, d \leq d_{\text {free }}$. In addition, $c_{d}$ is the total number of information bit errors produced by the incorrect paths of the Hamming weight.

Using $T(D, N)$, the upper bounds on the event error probability $P_{E}$ and the bit error probability $P_{B}$ of a code with rate $R_{i}=k / v$ are given as follows:

$$
\begin{aligned}
& P_{E} \leq \sum_{d=d_{\text {free }}}^{\infty} a_{d} P_{d} \\
& P_{B} \leq \frac{1}{k} \sum_{d=d_{\text {free }}}^{\infty} c_{d} P_{d} .
\end{aligned}
$$

Here,

$$
\begin{aligned}
& P_{d}=Q\left(\sqrt{2 d R \frac{E_{b}}{N_{0}}}\right) \\
& Q(x)=\int_{x}^{\infty} \frac{1}{2 \pi} \exp \left(-\frac{1}{2} z^{2}\right) d z .
\end{aligned}
$$

Here, $E_{b} / N_{0}$ is the energy per bit-to-noise density ratio and $P_{d}$ is the pairwise error probability in the case of

Table 1 Number of copies $n_{i}$ for each pattern under the AWGN channel

\begin{tabular}{lllllll}
\hline$i$ & 0 & 1 & 2 & 3 & 4 & $q=5$ \\
\hline Pattern $1, n_{i}$ & 1 & 1 & 1 & 1 & 2 & 4 \\
Pattern 2, $n_{i}$ & 1 & 2 & 2 & 4 & 4 & 6 \\
$R_{i}$ & $8 / 9$ & $4 / 5$ & $2 / 3$ & $1 / 2$ & $1 / 2$ & $1 / 2$ \\
\hline
\end{tabular}

Table 2 Number of copies $n_{i}$ for each pattern under the Rayleigh fading channel

\begin{tabular}{lllllll}
\hline$i$ & 0 & 1 & 2 & 3 & 4 & $q=5$ \\
\hline Pattern $1, n_{i}$ & 1 & 1 & 1 & 1 & 1 & 3 \\
Pattern $2, n_{i}$ & 1 & 3 & 4 & 4 & 5 & 5 \\
$R_{i}$ & $8 / 9$ & $4 / 5$ & $2 / 3$ & $1 / 2$ & $1 / 2$ & $1 / 2$ \\
\hline
\end{tabular}

PSK modulation and unquantized AWGN channels [25]. Then, in case of the Rayleigh fading channel, $P_{d}$ is expressed as follows [26]:

$$
\begin{aligned}
& P_{d}=\left(P_{e}\right)^{d} \sum_{t=0}^{d-1}\left(\begin{array}{c}
d-1-t \\
t
\end{array}\right)\left(1-P_{e}\right)^{t} \\
& P_{e}=1-\sqrt{\frac{\gamma_{b} R}{1+\gamma_{b} R}},
\end{aligned}
$$

where $\gamma_{b}$ is the average of $E_{b} / N_{0}$.

Further, $P_{i}$ is determined using $P_{B}$ from the following equation:

$$
P_{i}=1-\left(1-P_{B}\right)^{L_{i n f o}}
$$

Here, $L_{\text {info }}$ is the number of information bits. In addition, the upper bound of the residual bit error rate (RBER) is obtained by the following equation:

$$
\mathrm{RBER}=r_{B, q} \prod_{i=0}^{q-1} P_{i}
$$

Here, $r_{B, q}$ is $P_{B}$ in the case of a code with rate $R_{q}$. The transfer function $T(D, N)$ changes according to the punctured matrices. Further details of the transfer function $T(D, N)$ are provided in the Appendix.

Table 3 Simulation parameters

\begin{tabular}{ll}
\hline Parameter & Detail \\
\hline Channel model & AWGN \\
& Rayleigh fading \\
& IEEE802.15.6 CM3 \\
& BPSK \\
Modulation & $\mathrm{R}=8 / 9,4 / 5,2 / 3$ and $1 / 2$ \\
FEC & $\mathrm{K}=3$ convolutional codes \\
& Soft decision \\
Decoding & Viterbi decoding \\
& Weldon's ARQ \\
ARQ protocol & 504 bits \\
Linfo & $487 \mathrm{kbps}$ \\
Data rate & $9.84 \mathrm{~ms}$ \\
Roundtrip time &
\end{tabular}




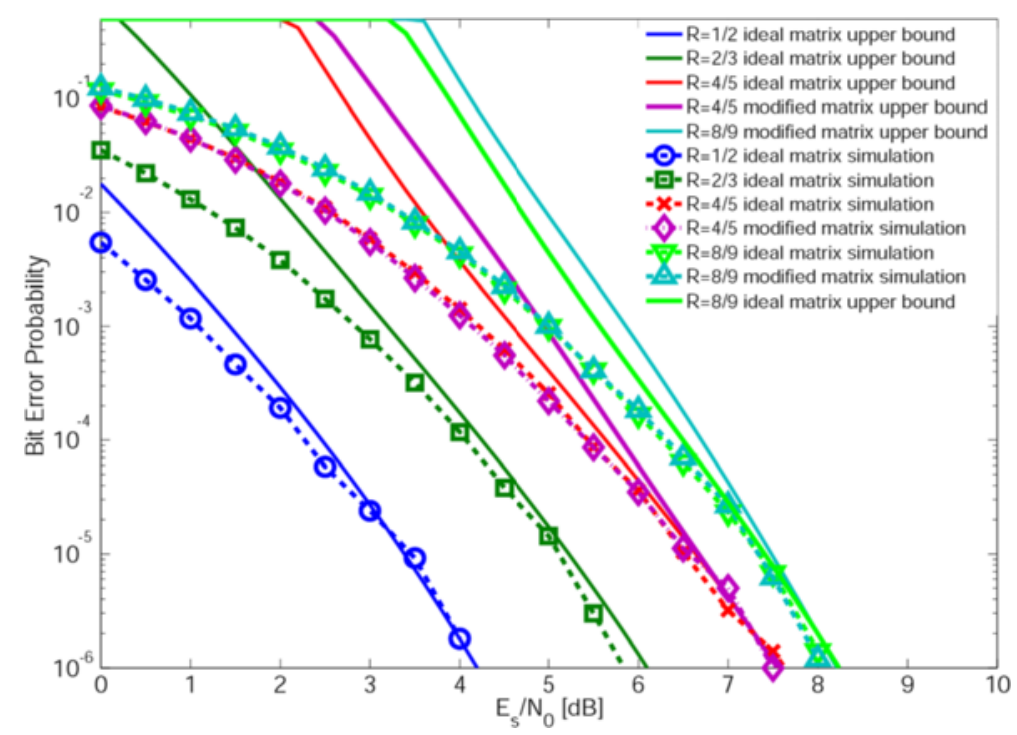

Fig. 6 Bit error probability of punctured convolutional codes under AWGN channel

\section{Performance evaluation}

\subsection{Simulation condition}

In this subsection, we evaluate our proposed scheme by theoretical analysis and simulations.

To analyze our proposed scheme, we set two patterns under the AWGN channel and the Rayleigh fading channel, as shown in Tables 1 and 2, respectively. A coding rate of a decomposable code $R_{i}$ is determined according to the order of Eq. 1. Then, $R_{i}$ is a coding rate after reconstructing a decomposable code. For the required QoS, the residual BER of pattern 1 is more important than its latency, and the latency of pattern 2 is more important than its residual BER. The number of copies of pattern 1 can achieve the maximum throughput in a high or middle $E_{s} / N_{0}$ area from our theoretical formulae. For pattern 2, the number of copies $n_{i}$ is large because the latency is allowed; however, the residual BER and throughput performance must be significantly improved. Also, pattern 2 is fixed, as the throughput of pattern 2 is higher than that of the high QoS mode defined in IEEE 802.15.6 (hereinafter called the conventional scheme).

Table 4 Optimal number of copies $n_{i}$ for minimum latency while satisfying PER $\leq 10^{-5}$. Channel model is the AWGN channel

\begin{tabular}{lllll}
\hline$E_{5} / N_{0}$ & $n_{0}$ & $n_{1}$ & $n_{2}$ & $n_{3}$ \\
\hline $3 \mathrm{~dB}$ & 1 & 1 & 10 & - \\
& 1 & 7 & $i=q$ & \\
$5 \mathrm{~dB}$ & 1 & $i=q$ & - & - \\
$6 \mathrm{~dB}$ & 1 & 4 & - & - \\
$R_{i}$ & $8 / 9$ & $4 / 5$ & $2 / 3$ & $1 / 2$ \\
\hline
\end{tabular}

Note that another channel model is assumed to be the Rayleigh fading channel, which is one of the channel models for wearable WBANs [27]. Then, IEEE802.15.6 CM3, which is a typical channel model for wearable WBANs [27], is also assumed in order to evaluate actual performance of our proposed system. This model suffers from strong multipath fading and shadowing. The data rate is referenced from the IEEE802.15.6 standard [17], and the roundtrip time is set based on the data rate and twice the maximum packet length defined in IEEE802.15.6. Simulation parameters are summarized in Table 3.

\subsection{Numerical results}

Figure 6 shows the bit error probability of the punctured convolutional codes under the AWGN channel. Our proposed punctured convolutional codes with coding rates of $4 / 5$ and $8 / 9$ have a different upper bound than that of the ideal matrix; however, these differences do not significantly influence the performance of our proposed scheme.

Relative to the simulation, the performance of the modified punctured convolutional codes is the same as

Table 5 Optimal number of copies $n_{i}$ for maximum throughput while satisfying PER $\leq 10^{-5}$. Channel model is the AWGN channel

\begin{tabular}{lllll}
\hline$E_{5} / N_{0}$ & $n_{0}$ & $n_{1}$ & $n_{2}$ & $n_{3}$ \\
\hline $3 \mathrm{~dB}$ & 1 & 1 & 1 & 3 \\
$5 \mathrm{~dB}$ & 1 & 1 & 2 & $i=9$ \\
$6 \mathrm{~dB}$ & 1 & 1 & 1 & - \\
$R_{i}$ & $8 / 9$ & $4 / 5$ & $2 / 3$ & - \\
\hline
\end{tabular}




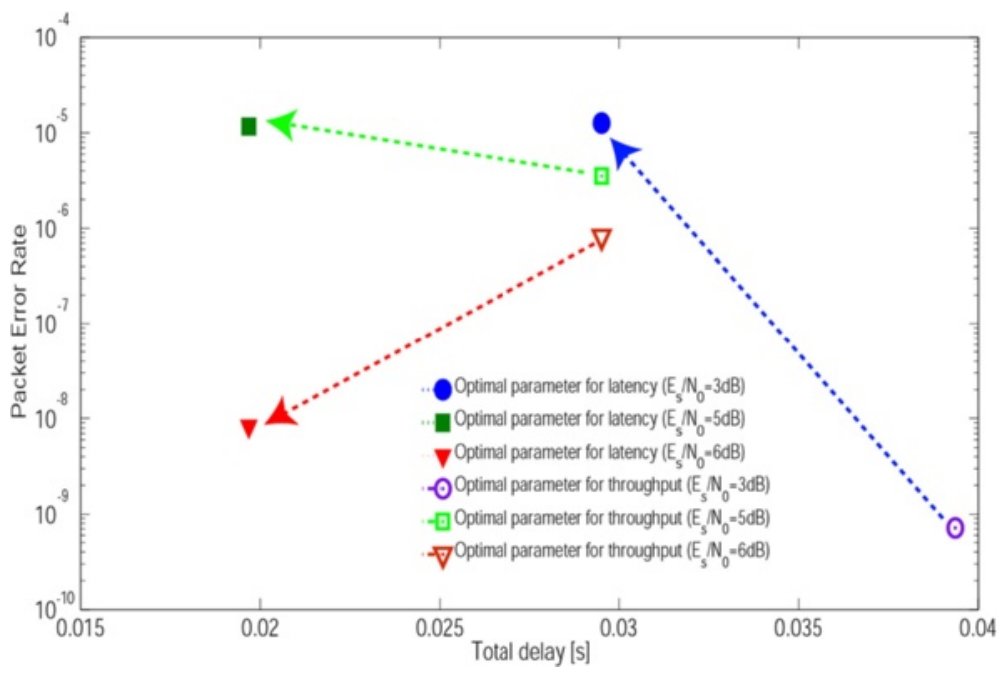

Fig. 7 PER performance with optimal and sub-optimal parameters under AWGN channel. Parameters of Table 4 are optimal

that of the ideal punctured matrices, unlike results of the upper bound. This occurs because the number of trials in the simulation is not large enough. Further, the difference between the error-correcting capacity of the ideal matrix and that of the modified one is very small in the simulation. Hence, the difference does not clearly emerge in the graph without the huge number of trials. The difference between the upper bound and the simulation becomes greater as $E_{s} / N_{0}$ decreases.

Table 4 shows examples of the optimal number of copies $n_{i}$ for the minimum latency while satisfying PER $\leq 10^{-5}$ by a full search under the AWGN channel. Table 5 shows examples of the optimal number of copies $n_{i}$ for the maximum throughput while meeting the same condition. Figures 7 and 8 show the theoretical PER and throughput, respectively, according to parameters of Tables 4 and 5 . The parameters of Table 4 satisfy the condition that PER $\leq 10^{-5}$ by a smaller delay than Table 5 in Fig. 7 , whereas those of Table 5 achieve a larger throughput than that of Table 4 even though a large delay is expected in Fig. 8. In other words, performance with non-optimal parameters in each policy can be considered the same as that of sub-optimal parameters. Then, Figs. 9 and 10 present throughput performances with parameters of Tables 4 and 5 as a function of $E_{s} / N_{0}$. From these figures, we observe that performance changes substantially depending on the parameters. Especially, Fig. 10 shows that each parameter achieves the maximum throughput with the smallest number of retransmissions and $n_{i}$ copies at each $E_{s} / N_{0}$.

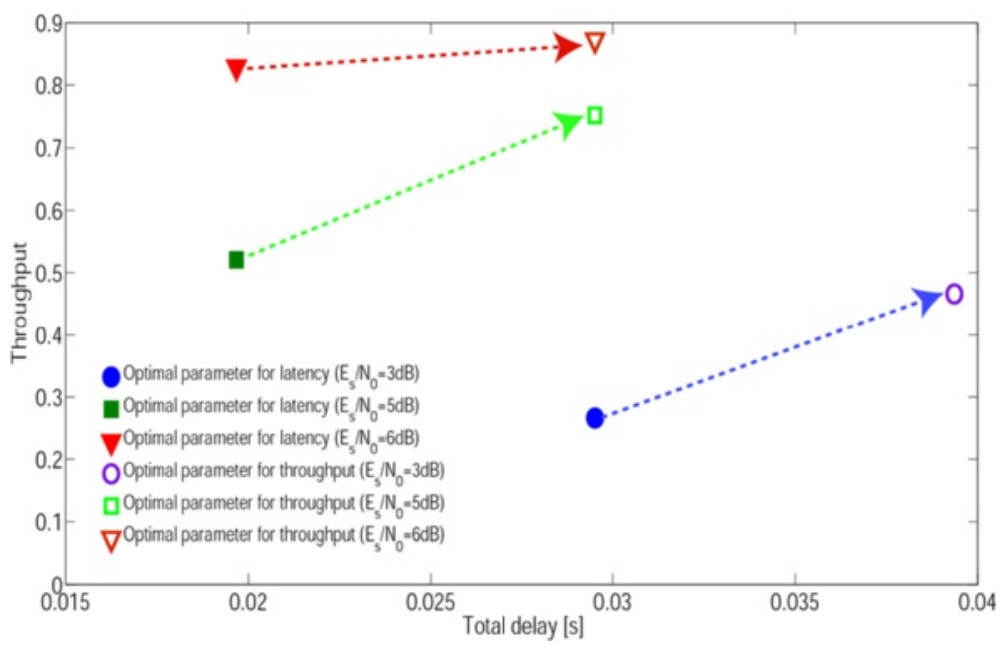

Fig. 8 Throughput performance with optimal and sub-optimal parameters under AWGN channel. Parameters of Table 5 are optimal 


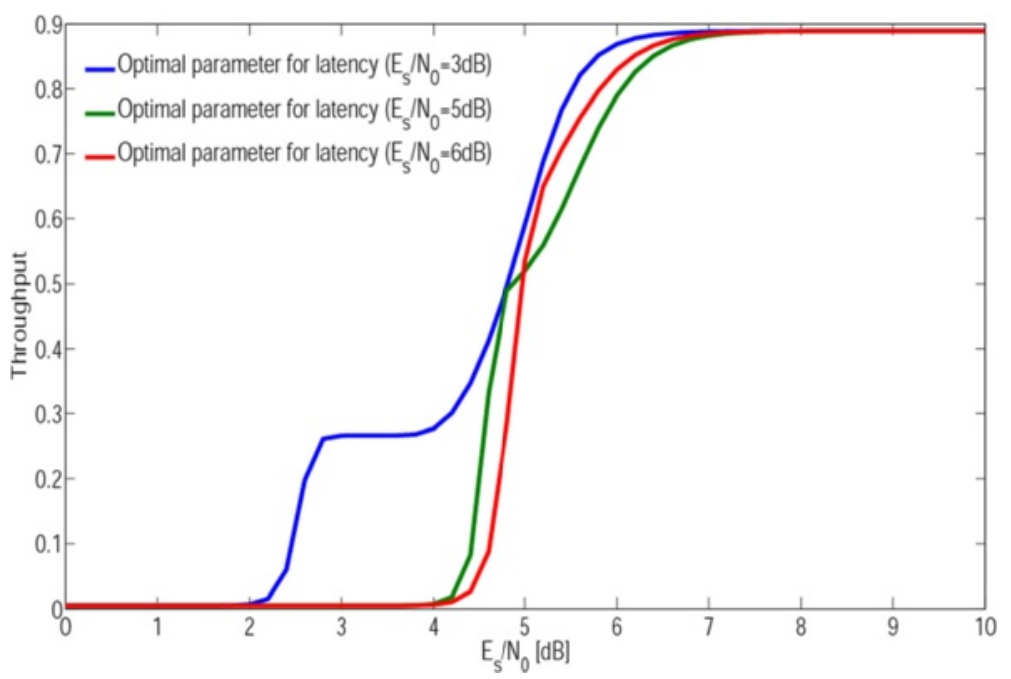

Fig. 9 Throughput performance with parameters of Table 4 under AWGN channel as a function of $E_{5} / N_{0}$

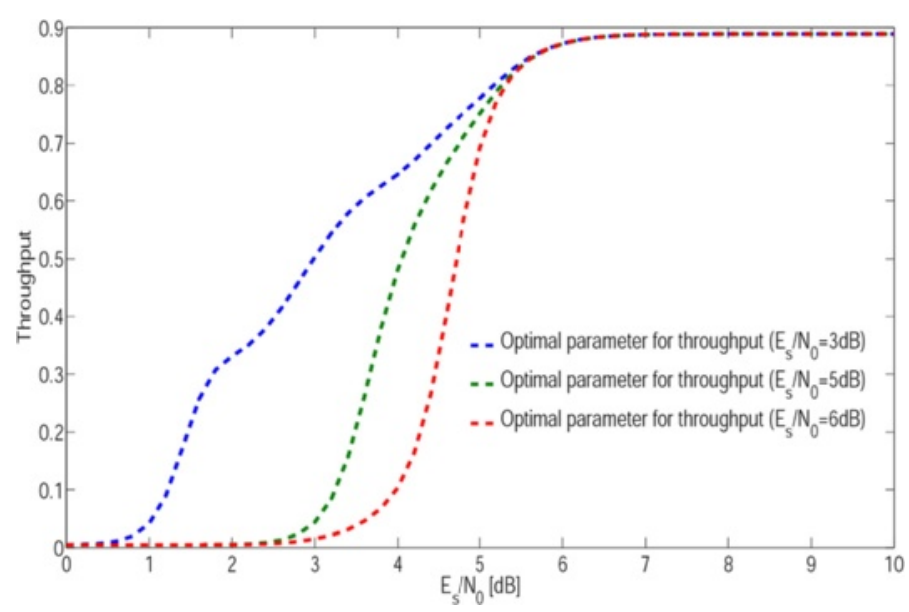

Fig. 10 Throughput performance with parameters of Table 5 under AWGN channel as a function of $E_{5} / N_{0}$

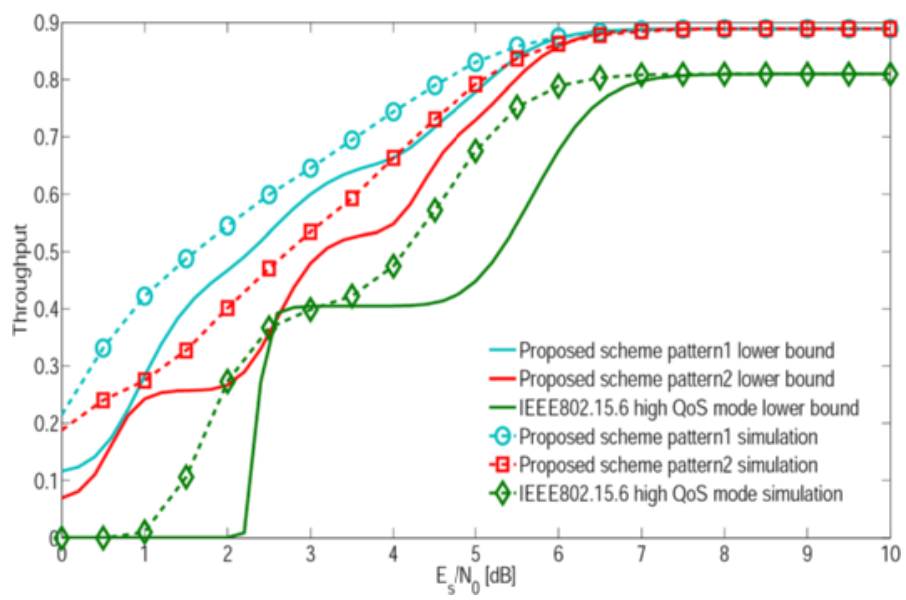

Fig. 11 Throughput performance in the proposed method and the conventional scheme under AWGN channel 


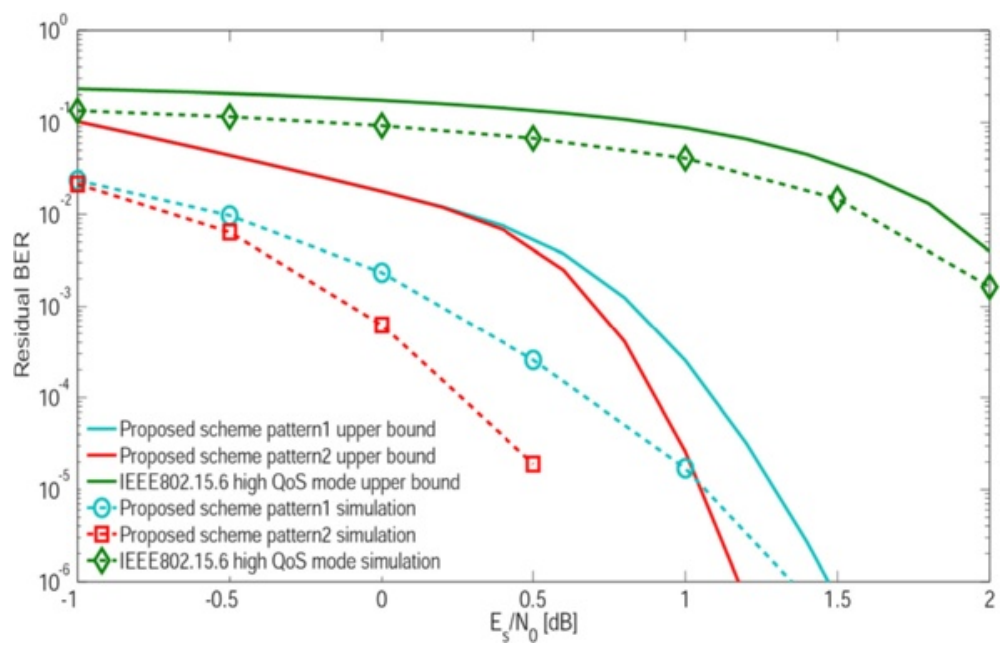

Fig. 12 Residual BER performance in the proposed and the conventional scheme under AWGN channel

Figures 11 and 12 show the throughput and residual BER performance, respectively, for patterns 1 and 2 of our proposed scheme and the conventional scheme under the AWGN channel. Overall, the residual BER performance of our proposed scheme is better than that of the conventional scheme. In the optional pattern, the proposed system obtains over $1.5 \mathrm{~dB}$ gain than IEEE802.15.6 system. The throughput of our proposed scheme is also better than that of the conventional scheme. Then, the gain of the proposed scheme is over $1.2 \mathrm{~dB}$ gain than that of IEEE802.15.6 scheme in the optional pattern. The throughput and residual BER performances of pattern 1 are opposite to those of pattern 2 because of the parameter settings shown in Table 1 . Note that simulation results differ from bounds shown in Fig. 6.

Table 6 shows examples of the optimal number of copies $n_{i}$ for the minimum latency while satisfying PER $\leq 10^{-5}$ by a full search under the Rayleigh fading channel. And then, Table 7 shows examples of the optimal number of copies $n_{i}$ for the maximum throughput while meeting the same condition. Figures 13 and 14 express the theoretical PER and throughput, respectively, depending on parameters of Tables 6 and 7. Selected $E_{s} / N_{0}$ is higher than that in the case of the AWGN channel. The reason is that the performance in case of the Rayleigh fading channel is worse than that of the AWGN case. The parameters of Table 6 achieve the condition that PER $\leq 10^{-5}$ by a smaller delay than Table 7 in Fig. 13, whereas those of Table 7 satisfy a larger throughput than Table 6 even though a large delay is needed in Fig. 14. Then, as the case of the AWGN channel, performance with non-optimal parameters in each policy can be regarded as that of sub-optimal parameters. Figures 15 and 16 also present throughput performances with parameters of Tables 6 and 7 as a function of $E_{s} / N_{0}$. From these figures, we can show that performance changes highly according to the parameters like the AWGN case. In particular, Fig. 16 shows that each parameter achieves the maximum throughput with the smallest number of retransmissions and $n_{i}$ copies at each $E_{s} / N_{0}$.

Figures 17 and 18 present the throughput and residual BER performance, respectively, for patterns 1 and 2 of our proposed scheme and the conventional scheme
Table 6 Optimal number of copies $n_{i}$ for minimum latency while satisfying PER $\leq 10^{-5}$. Channel model is the Rayleigh fading channel

\begin{tabular}{lllllll}
\hline$E_{5} / N_{0}$ & $n_{0}$ & $n_{1}$ & $n_{2}$ & $n_{3}$ & $n_{4}$ & $n_{5}$ \\
\hline $8 \mathrm{~dB}$ & 1 & 1 & 1 & 3 & - & - \\
& & & $i=q$ & & & \\
$11 \mathrm{~dB}$ & 1 & 1 & 4 & - & - & - \\
$14 \mathrm{~dB}$ & 1 & 7 & - & - & - & - \\
$R_{i}$ & $8 / 9$ & $4 / 5$ & $2 / 3$ & $1 / 2$ & $1 / 2$ & $1 / 2$ \\
\hline
\end{tabular}

Table 7 Optimal number of copies $n_{i}$ for maximum throughput while satisfying PER $\leq 10^{-5}$. Channel model is the Rayleigh fading channel

\begin{tabular}{lllllll}
\hline$E_{5} / N_{0}$ & $n_{0}$ & $n_{1}$ & $n_{2}$ & $n_{3}$ & $n_{4}$ & $n_{5}$ \\
\hline $8 \mathrm{~dB}$ & 1 & 1 & 1 & 1 & 1 & 1 \\
$11 \mathrm{~dB}$ & 1 & 1 & 1 & 1 & 1 & - \\
$14 \mathrm{~dB}$ & 1 & 1 & 1 & 1 & - & - \\
$R_{i}$ & $8 / 9$ & $4 / 5$ & $2 / 3$ & $1 / 2$ & $1 / 2$ & $1 / 2$ \\
\hline
\end{tabular}




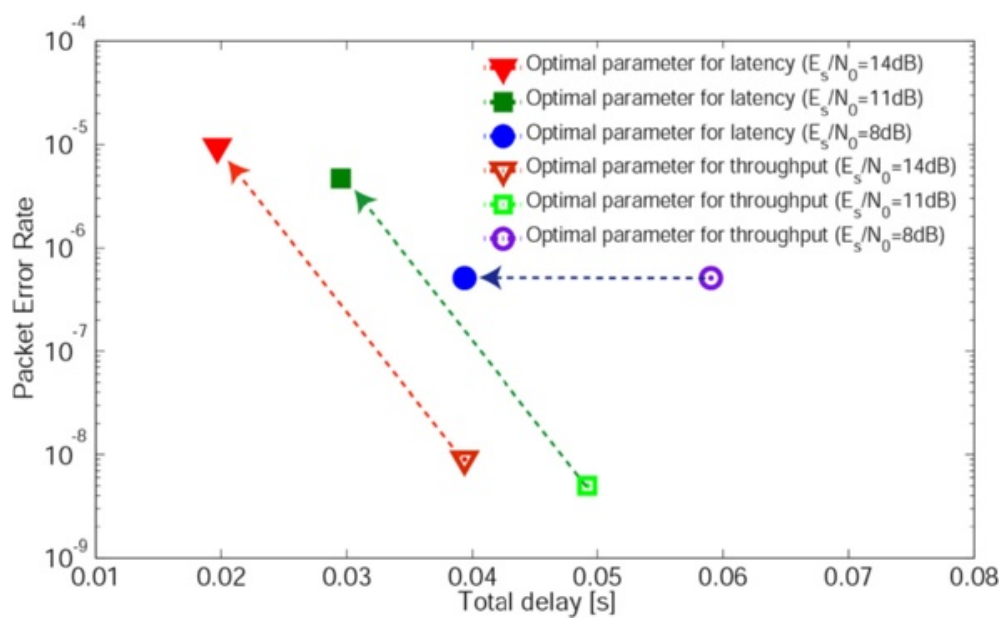

Fig. 13 PER performance with optimal and sub-optimal parameters under the Rayleigh fading channel. Parameters of Table 6 are optimal

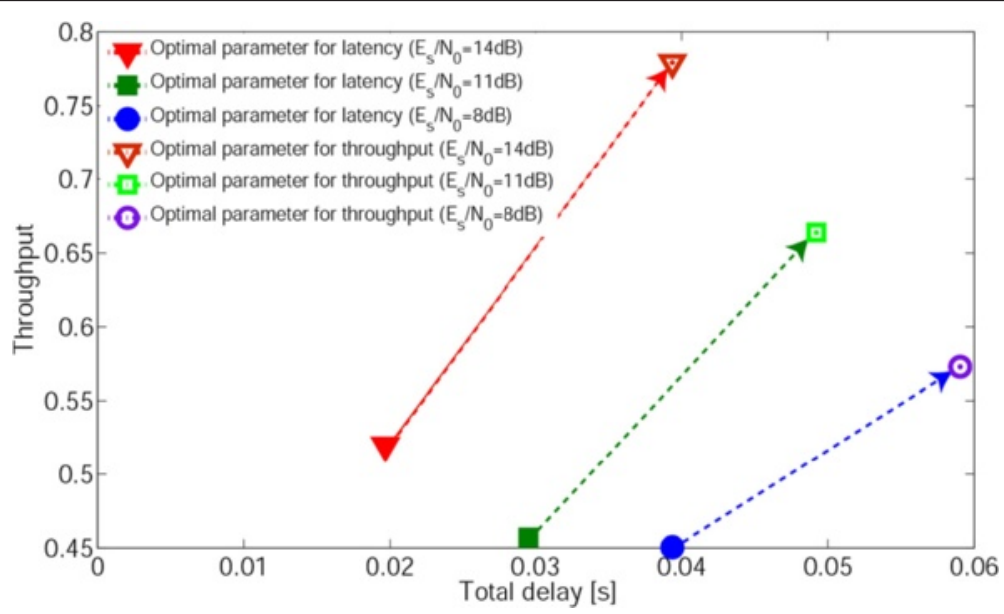

Fig. 14 PER performance with optimal and sub-optimal parameters under the Rayleigh fading channel. Parameters of Table 7 are optimal

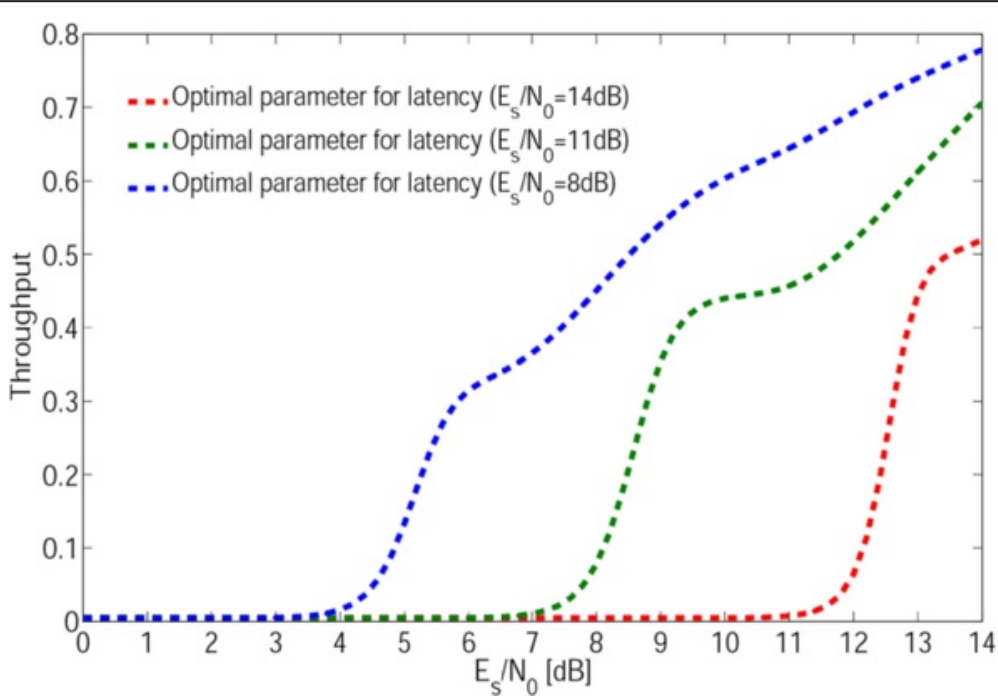

Fig. 15 Throughput performance with parameters of Table 6 under the Rayleigh fading channel as a function of $E_{s} / N_{0}$ 


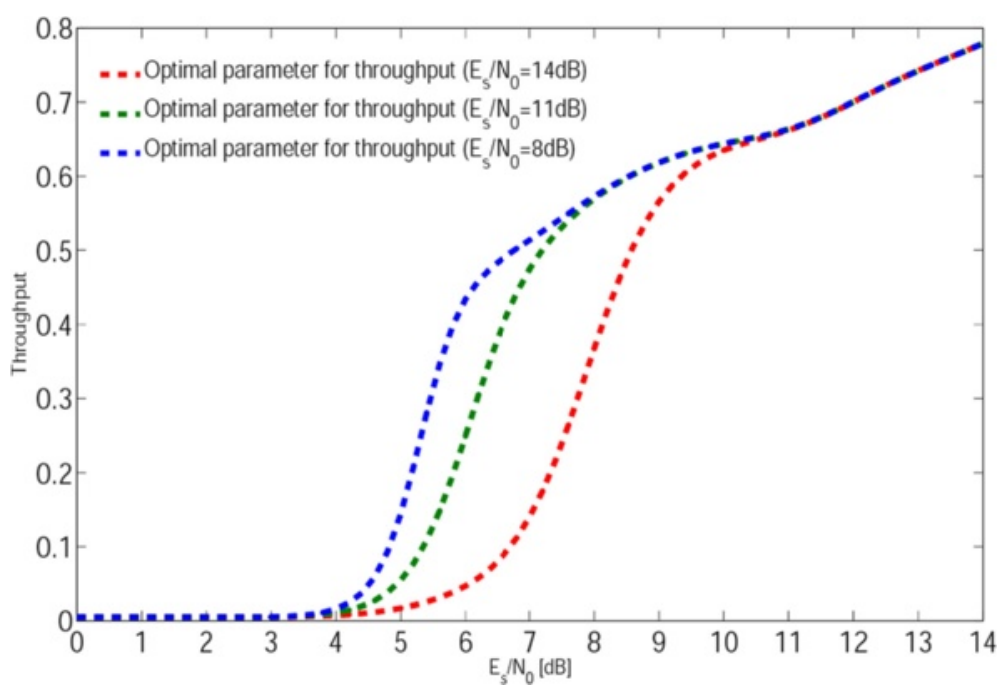

Fig. 16 Throughput performance with parameters of Table 7 under the Rayleigh fading channel as a function of $E_{5} / N_{0}$

under the Rayleigh fading channel. On the whole, our proposed scheme has better residual BER performance than the conventional scheme. In the optional pattern, the proposed system obtains over $4.2 \mathrm{~dB}$ gain than the standard system. The throughput of our proposed scheme is also better than that of the conventional scheme. Then, the gain of the proposed scheme is over $4.5 \mathrm{~dB}$ gain than that of IEEE802.15.6 in the optional pattern. The throughput and residual BER performances of pattern 1 are also opposite to those of pattern 2 because of the parameter settings shown in Table 2 like the AWGN case. Note that simulation results are different from bounds for the same reason as the case of the AWGN channel.
Figures 19 and 20 show the throughput and residual BER performance, respectively, for two patterns of our proposed scheme and the conventional scheme in the IEEE802.15.6 CM3 channel using simulations as examples of actual performance in a realistic environment. The reason is that it is difficult to theoretically analyze optimal parameters of these schemes in this channel model because the channel model is made by an experiment. Here, parameters are set based on Table 1 as a concrete example. In this channel model, the performance of our proposed scheme is better than that of the conventional scheme. Compared with the AWGN channel and the Rayleigh fading channel, however, the

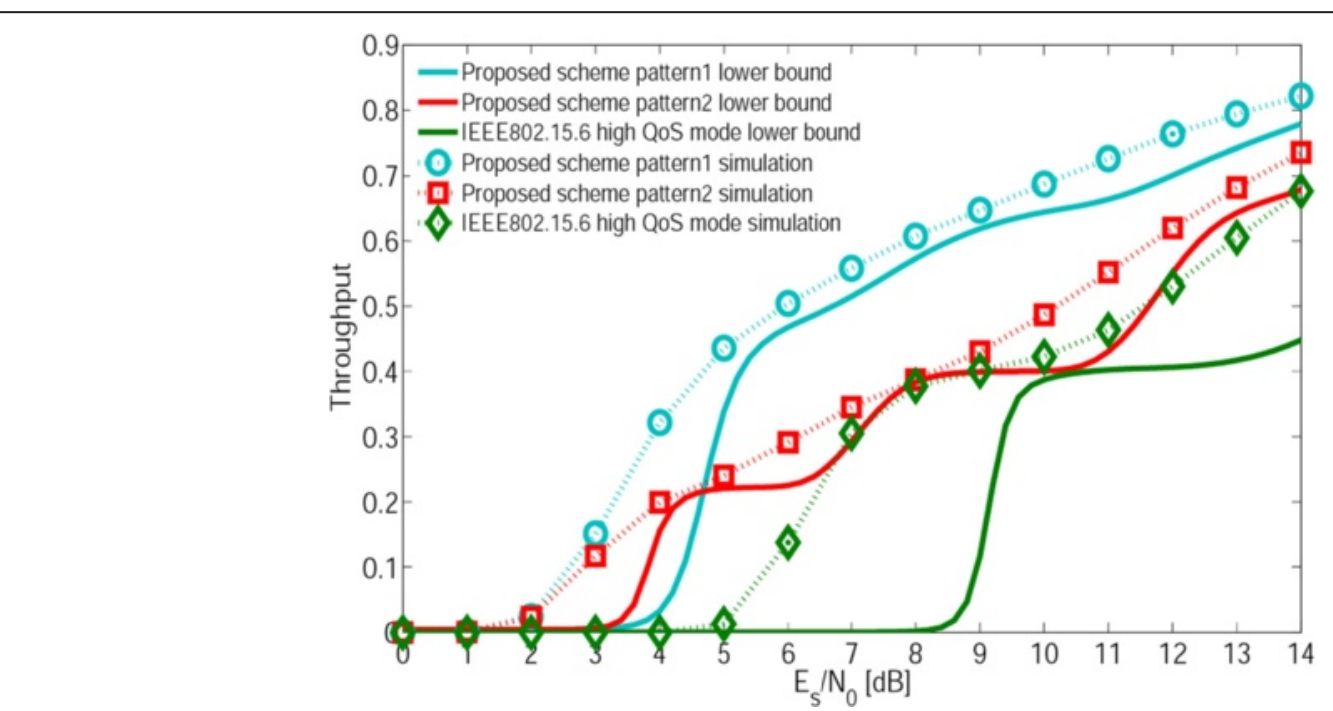

Fig. 17 Throughput performance in the proposed method and the conventional scheme under the Rayleigh fading channel 


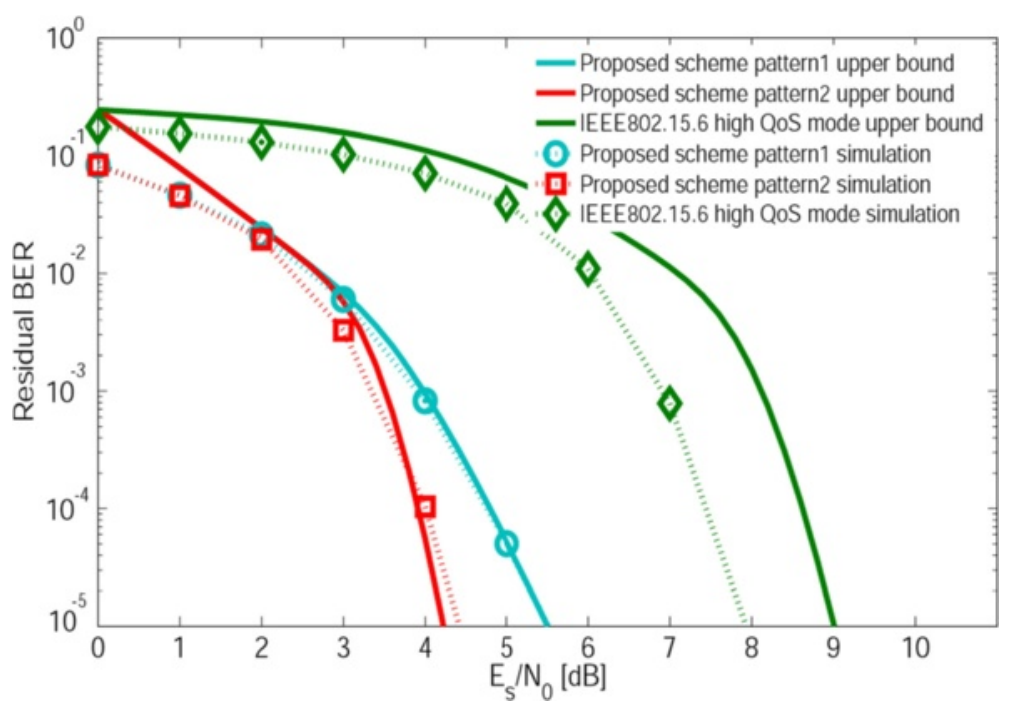

Fig. 18 Residual BER performance in the proposed and the conventional scheme under the Rayleigh fading channel

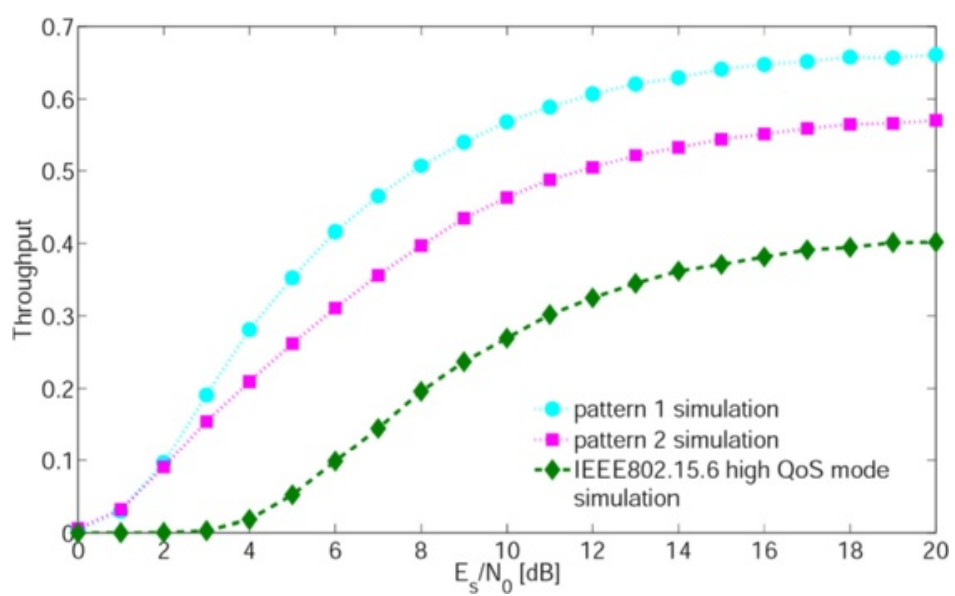

Fig. 19 Throughput performance in proposed and conventional schemes under IEEE802.15.6 CM3

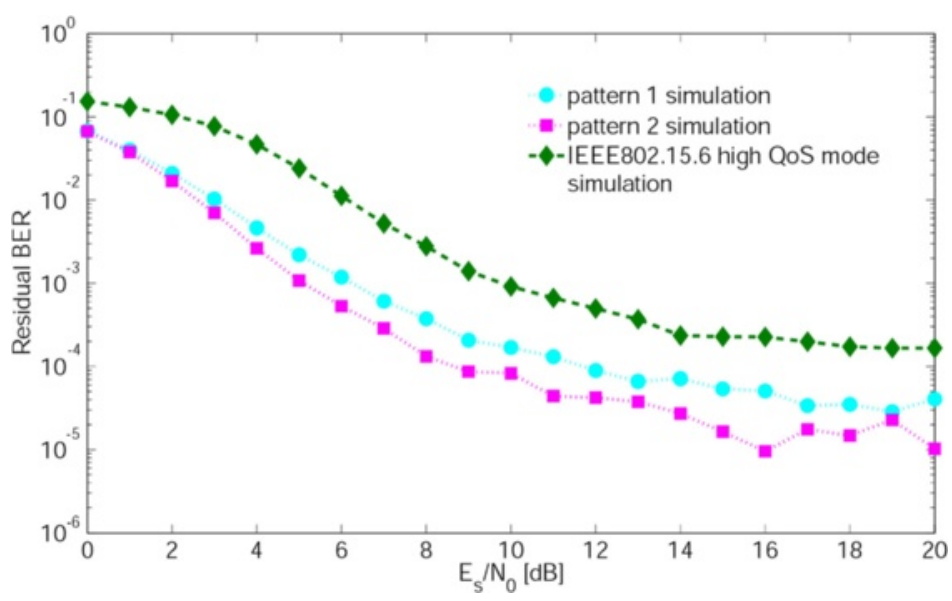

Fig. 20 Residual BER performance in proposed and conventional schemes under IEEE802.15.6 CM3 
performance is worse because of the strong multipath fading or shadowing. For this reason, the difference in the performance of our proposed and the conventional schemes is not larger than that of the AWGN channel; however, the tendency of the performance is the same as that of the AWGN channel and the Rayleigh fading channel.

\section{Conclusions}

In this paper, we presented a theoretical analysis of our proposed scheme to evaluate it under the AWGN channel and the Rayleigh fading channel as an extension of our previous work. We investigated QoS parameters to further optimize our system, showing that our proposed system can achieve optimal performance by arbitrarily selecting parameters of the error-correcting code and Hybrid ARQ. Performance evaluations by theoretical analysis and simulations showed that our proposed scheme has greater flexibility for optimizing QoS parameters according to the required QoS for each input data. Moreover, our evaluations showed that the performance of our proposed scheme is better than that of the conventional scheme.

In the future, we will consider a dynamic channel model and situation such as a multiple WBAN environment that can be theoretically analyzed. Then, optimal parameters for that case should also be investigated.

\section{Appendix}

\subsection{Transfer function}

To obtain $P_{E}$ and $P_{B}$, we derive the transfer function $T(D, N)$ from the state diagram using the transfer function technique $[24,25]$. Figure 21 shows the state diagram for the punctured convolutional codes.
Here, $X_{a}$ and $X_{b}$ express state zero, and the remaining states are arbitrarily labeled $X_{1}, X_{2}$, and $X_{3}$. The exponent of $N$ indicates the number of information bits set to "1" that causes the transition to occur, and the exponent of $D$ indicates the Hamming weight on the transition. From Fig. 21, the matrix equation is given as follows:

$$
\begin{aligned}
{\left[\begin{array}{l}
X_{1} \\
X_{2} \\
X_{3}
\end{array}\right]=} & {\left[\begin{array}{lll}
g_{11} & g_{12} & g_{13} \\
g_{21} & g_{22} & g_{23} \\
g_{31} & g_{32} & g_{33}
\end{array}\right]\left[\begin{array}{l}
X_{1} \\
X_{2} \\
X_{3}
\end{array}\right] } \\
& +\left[\begin{array}{c}
\left(N^{3}+N^{2}+N\right) D^{2}+N^{2} D^{4} \\
\left(N^{2}+N\right) D+N^{3} D^{3}+N^{2} D^{5} \\
N^{2} D+\left(N^{4}+2 N^{3}\right) D^{3}
\end{array}\right] X_{a}
\end{aligned}
$$

$$
\left\{\begin{array}{c}
g_{11}=N^{3}+N^{2} D^{2}+\left(N^{2}+N\right) D^{4} \\
g_{12}=\left(N^{3}+2 N^{2}\right) D^{2}+N D^{4} \\
g_{13}=\left(N^{3}+2 N^{2}\right) D^{2}+N D^{4} \\
g_{21}=N^{3} D+\left(2 N^{2}+N\right) D^{3} \\
g_{22}=N^{2} D+\left(N^{3}+N^{2}+N\right) D^{3} \\
g_{23}=N^{2} D+\left(N^{3}+N^{2}+N\right) D^{3} \\
g_{31}=N^{7} D+N^{3} D^{3}+N^{2} D^{5} \\
g_{32}=N^{3} D+\left(N^{4}+N^{3}+N^{2}\right) D^{3} \\
g_{33}=N^{3} D+\left(N^{4}+N^{3}+N\right) D^{3}
\end{array}\right.
$$

$$
\begin{aligned}
\boldsymbol{X}= & \boldsymbol{A} \boldsymbol{X}+\boldsymbol{F} X_{a} \\
X_{b}= & {\left[\begin{array}{c}
\left(N^{2}+N+1\right) D^{2}+N D^{4} \\
\left(2 N^{2}+1\right) D^{2}+N^{2} D^{4} \\
\left(2 N^{2}+1\right) D^{2}+N^{2} D^{4}
\end{array}\right]{ }^{T}\left[\begin{array}{l}
X_{1} \\
X_{2} \\
X_{3}
\end{array}\right] } \\
& +\left\{N^{2} D^{2}+\left(N^{2}+N\right) D^{4}\right\} X_{a}
\end{aligned}
$$

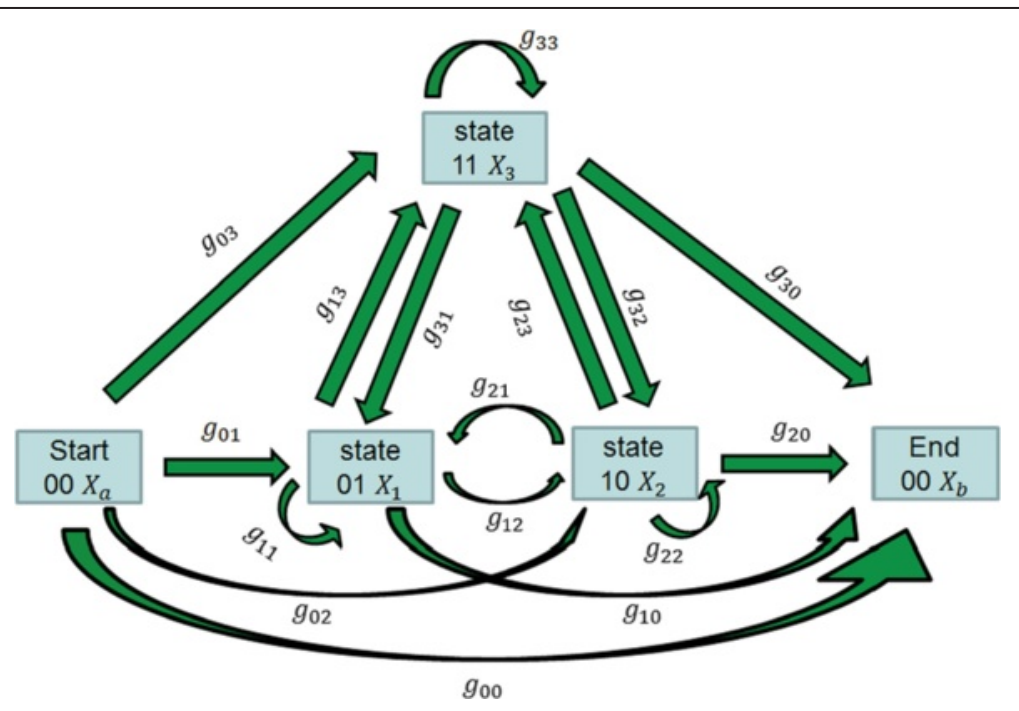

Fig. 21 State diagram of punctured convolutional codes 


\section{Competing interests}

The authors declare that they have no competing interests.

\section{Acknowledgements}

The first author would like to thank members of Kohno laboratory, Yokohama National University, Japan for their great inspiration and kindness.

\section{Author details}

'Graduate School of Engineering, Yokohama National University, 79-5 Tokiwadai, Hodogaya-Ku, 240-8501 Yokohama, Japan. ${ }^{2}$ Graduate School of Information Sciences, Hiroshima City University, 3-4-1, Ozuka-Higashi, Asa-Minami-Ku, 731-3194 Hiroshima, Japan.

Received: 6 September 2015 Accepted: 18 February 2016 1. 0

\section{References}

1. M Chen, S Gonzalez, A Vasilakos, H Cao, VCM Leung, Body area networks: a survey. Mobile Networks and Applications, Springer 16(2), 171-193 (2010)

2. G Acampora, DJ Cook, P Rashidi, AV Vasilakos, A survey on ambient intelligence in healthcare. Proc. IEEE 101(12), 2470-2494 (2013)

3. T Hayajneh, G Almashaqbeh, S Ullah, AV Vasilakos, A survey of wireless technologies coexistence in WBAN: analysis and open research issues. Wireless Networks, Springer 20(8), 2165-2199 (2014)

4. G Fortino, GD Fatta, M Pathan, AV Vasilakos, Cloud-assisted body area networks: state-of-the-art and future challenges. Wireless Networks, Springer 20(7), 1925-1938 (2014)

5. J Zhou, Z Cao, X Dong, X Lin, AV Vasilakos, Securing m-healthcare social networks: challenges, countermeasures and future directions. Wireless Communications, IEEE 20(4), 12-21 (2013)

6. J Zhou, Z Cao, X Dong, N Xiong, AV Vasilakos, 4S: a secure and privacypreserving key management scheme for cloud-assisted wireless body area network in m-healthcare social networks. Information Sciences, Elsevier 314(2015), 255-276 (2014)

7. D He, C Chen, S Chan, J Bu, AV Vasilakos, ReTrust: attack-resistant and lightweight trust management for medical sensor networks. Transactions on Information Technology in Biomedicine, IEEE 16(4), 623-632 (2012)

8. Z Zhang, H Wang, AV Vasilakos, H Fang, ECG-cryptography and authentication in body area networks. Transactions on Information Technology in Biomedicine, IEEE 16(6), 1070-1078 (2012)

9. D Lin, F Labeau, AV Vasilakos, QoE-based optimal resource allocation in wireless healthcare networks: opportunities and challenges. Wireless Networks, Springer 21(8), 2483-2500 (2015)

10. H Cao, V Leung, C Chow, H Chan, Enabling technologies for wireless body area networks: a survey and outlook. IEEE Comm. Mag. 47(12), 84-93 (2009)

11. H Viswanathan, B Chen, D Pompili, Research challenges in computation, communication, and context awareness for ubiquitous healthcare. IEEE Comm. Mag. 50(5), 92-99 (2012)

12. C Bachmann, M Ashouei, V Pop, M Vidojkovic, HD Groot, B Gyselinckx, Lowpower wireless sensor nodes for ubiquitous long-term biomedical signal monitoring. IEEE Comm. Mag. 50(1), 20-27 (2012)

13. JMLP Caldeira, JJPC Rodrigues, P Lorenz, Toward ubiquitous mobility solutions for body sensor networks on healthcare. IEEE Comm. Mag. 50(5), 108-115 (2012)

14. A Boulis, D Smith, D Miniutti, L Libman, Y Tselishchev, Challenges in body area networks for healthcare: the MAC. IEEE Comm. Mag. 50(5), 100-106 (2012)

15. A Wong, M Dawkins, G Devita, N Kasparidis, A Katsiamis, O King, F Lauria, J Schiff, A Burdett, A IV 5 mA Multimode IEEE 802.15.6/Bluetooth Low-Energy WBAN Transceiver for Biotelemetry Applications. Proceedings of Solid-State Circuits Conference Digest of Technical Papers (ISSCC), 2012 IEEE International, 2012, pp. 300-302

16. T Suzuki, H Tanaka, S Minami, H Yamada, T Miyata, Wearable wireless vital Monitoring Technology for Smart Health Care. Proceedings of 7th International Symposium on Medical Information and Communication Technology (ISMICT): March 2013; Tokyo, Japan, 2013

20. IEEE Standard for Information technology - Telecommunications and information exchange between systems - Local and metropolitan area networks- Specific requirements: Part 15.6: Wireless Medium Access Control (MAC) and Physical Layer (PHY) Specifications for Wireless Personal Area Networks (WPANs) used in or 12 around a body. IEEE (2011)
18. IEEE Standard for Local and metropolitan area networks Part 15.4: Low-Rate Wireless Personal Area Networks (LR-WPANs), Amendment: Alternative Physical Layer Extension to support Medical Body Area Networks (MBANS) services operating in the $2360-2400 \mathrm{MHz}$ band. IEEE (2013)

20. ETSI TC Smart BAN: SmartBAN System Description. TR DTS/SmartBAN-008 V0.1.0 (2014)

20. K Takabayashi, H Tanaka, C Sugimoto, R Kohno, An Error Control Scheme with Weldon's ARQ Considering Various QoS in Medical and Non-medical Uses for Wireless BANs. Proceedings of 7th International Symposium on Medical Information and Communication Technology (ISMICT): March 2013; Tokyo, Japan, 2013

21. K Takabayashi, H Tanaka, C Sugimoto, R Kohno, Multiplexing and Error Control Scheme with Modified Hybrid ARQ for Body Area Network employing IEEE 802.15.6 in UWB-PHY. Proceedings of 8th International Conference on Body Area Networks (BodyNets 2013): September 2013; Boston, Massachusetts, United States, 2013

22. K Takabayashi, H Tanaka, C Sugimoto, R Kohno, Multiplexing and Error Control Scheme for Body Area Network employing IEEE 802.15.6. Transactions on Communications, IEICE E97-B(03), 2014, pp. 564-570

25. E Weldon Jr, An improved selective-repeat ARQ strategy. IEEE Trans. Comm. 30(3), 480-486 (1982)

24. AJ Viterbi, Convolutional codes and their performance in communication systems. Transactions on Communication Technology, IEEE 19(5), 751-772 (1971)

25. D Haccoun, G Begin, High-rate punctured convolutional codes for viterbi and sequential decoding. IEEE Trans. Comm. 37(11), 1113-1125 (1989)

26. P Franger, P Orten, T Ottosson, Convolutional codes with optimum distance spectrum. IEEE Commun. Lett. 3(11), 317-319 (1999)

27. KY Yazdandoost, K Sayrafian-Pour, Channel Model for Body Area Network (BAN). IEEE P802.15 Working Group for Wireless Per-sonal Area Networks(WPANs), IEEE P802.15-08-0780-10-0006, 2009

\section{Submit your manuscript to a SpringerOpen ${ }^{\circ}$ journal and benefit from:}

- Convenient online submission

- Rigorous peer review

Immediate publication on acceptance

- Open access: articles freely available online

- High visibility within the field

- Retaining the copyright to your article

Submit your next manuscript at $\gg$ springeropen.com 